\title{
Stabilisation of a class of stochastic bilinear interconnected systems by suboptimal decentralised feedback controls
}

\author{
K PATRALEKH and R SINGH \\ Department of Electrical Engineering, Bhagalpur College of Engineering, \\ Bhagalpur 813210 , India \\ MS received 1 April 1998
}

\begin{abstract}
A computationally simple method has been suggested for decentralised stabilisation of a class of stochastic bilinear interconnected systems by optimal feedback controls at subsystem levels, appropriately designated "Suboptimal Decentralised Feedback Control". The proposed method makes use of an aggregation--decomposition technique based on algebraic Riccati eqations. The method has been illustrated through a numerical example of the stochastic bilinear interconnected system consisting of three subsystems, each of second order.
\end{abstract}

Keywords. Stochastic bilinear system; decentralised stabilisation; optimal feedback control.

\section{Introduction}

The problem of decentralised stabilisation of both linear and bilinear interconnected subsystems has been studied earlier by Siljak \& Vukcevic (1977) who have suggested a method for decentralised stabilisation by using centralised stability conditions. The authors obtain the comparison system by using the vector Liapunov function-based aggregation technique. The desired stabilising local control gain vectors are then computed by applying determinantal inequality tests to the aggregate matrix of the comparison system. Moreover, for obtaining the comparison system in case of bilinear interconnected system, the similarity transformation is applied so that the coefficient matrices of the interaction-free parts are transformed to the diagonal form and suitable bounds are used for the transformed interaction terms. Hence, the method is computationally involved.

The present work has also considered the $N$ interconnected bilinear subsystems as in the literature (Siljak \& Vukcevic 1977) but with white-noise parameter perturbations of the elements of the subsystem matrices resulting in $N$ stochastic bilinear interconnected subsystems. The aim of the present work is to extend the aggregation-decomposition technique based on algebraic Riccati equations developed in the literature (Patralekh \& Singh 1997 ) for $N$ linear interconnected subsystems to the $N$ stochastic bilinear interconnected 
subsystems. This is used for decentralised stabilisation so that the interconnected subsystems are asymptotically stable with probability one (ASwp1). The procedure of Mahalanabis \& Purkayastha (1973) based on the definition of ASwp1 has also been used for developing the aggregation-decomposition technique. The interaction-free and noise-free part of each subsystem is linear and time-invariant. The interaction terms are bilinear. The terms arising out of the white-noise parameter perturbations represent the state-dependent noise. It is assumed that the state vectors of the subsystems are bounded (Siljak \& Vukcevic 1977). This assumption is used for bounding the terms involving interactions for aggregation-decomposition. It is known that the optimal feedback control for a linear time-invariant system which minimises the quadratic performance criterion is the best stabilising control. Hence, the interaction-free and noise-free parts of the subsystems are first stabilised by the optimal feedback controls generated from the solution of the $N$ algebraic Riccati equations resulting from the optimal control problem. The $N$ algebraic Riccati equations are solved by a computationally simple method (Singh 1970). This requires the solution of only $\left(n_{i}-1\right)$ nonlinear algebraic equations by the Newton-Raphson method for subsystems of order $n_{i}$. Then, by using the aforesaid aggregation-decomposition technique, the maximum possible effects of interactions and state-dependent noise are incorporated into the coefficient matrix of the interaction-free and noise-free part of a given subsystem resulting in $N$ decoupled subsystems. Additional controls can then be applied to annul the effects of the interactions so that the decoupled subsystems have the same locations as the eigenvalues and are ASwp1 with the same degree of stability and optimal response as the corresponding interaction-free and noise-free parts stabilised with optimal controls. Thus the desired stabilisation is achieved with the advantage that the response is optimal. Since the optimal controls minimise the quadratic performance criteria, the cost of control energy is also minimum.

The decentralised stabilising controls which are optimal at subsystems level are designated "Suboptimal Decentralised Feedback Controls" for the composite system.

\section{Problem formulation}

Consider an interconnected bilinear differential system modelled by the following state equations (Siljak \& Vukcevic 1977) but having white-noise parameter perturbations of the elements of the subsystem matrices,

$$
\begin{gathered}
\dot{x_{i}}=A_{i i} x_{i}+b_{i} u_{i}+a_{i} x_{l}^{T} \sum_{j=1, j \neq i, l}^{N} A_{i, j} x_{j}, \\
i=1,2, \ldots, N ; \quad l= \begin{cases}1, & i=N, \\
i+1, & i \neq N,\end{cases}
\end{gathered}
$$

where $x_{i} \triangleq\left[x_{i 1} x_{i 2} \cdots x_{i n_{i}}\right]^{T}$ and $u_{i}$ is the scalar control.

The coefficient matrices $A_{i i}$ and $A_{i j}$ have $a_{i i w l}, a_{i j w w^{\prime}}$ as the $w, l$ th, $w, w^{\prime}$ th elements, $i, j=1,2, \ldots, N ; w, l=1,2, \ldots, n_{i} ; w^{\prime}=1,2, \ldots, n_{j}$. The elements $a_{i i w l}, a_{i j w w^{\prime}}$ are 
assumed to have the form,

$$
\begin{aligned}
a_{i i w l} & =a_{i i w l}^{0}+\sum_{v=1}^{n_{i}} e_{i i w l v} \dot{w}_{i v}, \\
a_{i j w w^{\prime}} & =a_{i j w w^{\prime}}^{0}+\sum_{v^{\prime}=1}^{n_{j}} e_{i j w w^{\prime} v^{\prime}} \dot{w}_{j v^{\prime}},
\end{aligned}
$$

$a_{i i w l}^{0}, a_{i j w w^{\prime}}^{0}$ being the nominal values and $\sum_{v=1}^{n_{i}} e_{i i w l v} \dot{w}_{i v}, \sum_{v^{\prime}=1}^{n_{j}} e_{i j w w^{\prime} v^{\prime}} \dot{w}_{j v^{\prime}}$ the additive white-noise perturbations. Thus the state equations (1) can be rewritten as

$$
\begin{aligned}
\dot{x}_{i}=A_{i i}^{0} x_{i}+b_{i} u_{i}+a_{i} x_{l}^{T} \sum_{j=1, j \neq i, l}^{N} A_{i j}^{0} x_{j} \\
+E_{i i}\left(x_{i}\right) \dot{w}_{i}+\sum_{j=1, j \neq i, l}^{N} a_{i} x_{l}^{T} E_{i j}\left(x_{j}\right) \dot{w}_{j}
\end{aligned}
$$

where $A_{i i}^{0} x_{i}$ is the autonomous part of the interaction-free and noise-free terms. The summation terms $a_{i} x_{l}^{T} \sum_{j=1, j \neq i, l}^{N} A_{i j}^{0} x_{j}$ represent the effects of interactions among the $i$ th and other subsystems.

The terms $E_{i i}\left(x_{i}\right) \dot{w}_{i}$ and $\sum_{j=1, j \neq i, l}^{N} a_{i} x_{l}^{T} E_{i j}\left(x_{j}\right) \dot{w}_{j}$ represent the state dependent noise. $w_{j}$ is taken as $n_{j} \times 1$ dimensional Wiener process so that $\dot{w}_{j}$ is white and Gaussian. The state dependent matrix $E_{i i}\left(x_{i}\right)$ has $\sum_{v=1}^{n_{j}} e_{i i w v l} x_{i v}$ as the $w, l$ th element. The state dependent matrix $E_{i j}\left(x_{j}\right)$ has $\sum_{v^{\prime}=1}^{n_{j}} e_{i j w v^{\prime} w^{\prime}} x_{j v^{\prime}}$ as the $w, w^{\prime}$ th element. It is assumed that $\left(A_{i i}^{0}, b_{i}\right)$ is in the companion form,

$$
\Rightarrow A_{i i}=\left[\begin{array}{cccccc}
0 & 1 & 0 & 0 & \ldots & 0 \\
0 & 0 & 1 & 0 & \ldots & 0 \\
. & . & . & . & \ldots & . \\
. & . & . & . & \ldots & . \\
0 & 0 & 0 & \ldots & 0 & 1 \\
-c_{i 1} & -c_{i 2} & -c_{i 3} & \ldots & . & -c_{i n_{i}}
\end{array}\right], \quad b_{i}=\left[\begin{array}{lll}
0 & 0 & 0 \\
0 & \cdots & b_{i n_{i}}
\end{array}\right]^{T}
$$

It is further assumed that $\left(A_{i i}^{0}, b_{i}\right)$ is completely controllable. In the interaction term $A_{i j}^{0}$ is an $n_{i} \times n_{j}$ real matrix and $a_{i}=\left[\begin{array}{ll}a_{i 1} & a_{i 2}, \ldots, a_{i n_{i}}\end{array}\right]^{T}$. As in the literature (Siljak \& Vukcevic 1977), it is assumed that

$$
\begin{aligned}
& \left\|x_{i}\right\| \leq U_{i}^{0}, \quad i=1,2, \ldots, N, \\
& \Rightarrow\left\|x_{l}\right\| \leq U_{l}^{0}, \quad l=1,2, \ldots, N,
\end{aligned}
$$

where $U_{l}^{0}$ is a positive constant.

Now the problem at hand is to find the state feedback gain vector $K_{i}$ for generating the decentralised feedback control,

$$
u_{i}=K_{i} x_{i}, \quad i=1,2, \ldots, N
$$


for each of the $i$ th subsystem of (2) such that the composite system is ASwp1. It is further desired that the $i$ th subsystem is ASwp1 with the same degree of stability and optimal response as the corresponding interaction-free and noise-free part when stabilised with optimal control.

\section{Optimal feedback control for the interaction-free and noise-free part}

It is know that the optimal feedback control $u_{i}^{*}$ for the interction-free and noise-free part of the $i$ th subsystem of (2) which minimises the quadratic performance criterion,

$$
I=\int_{0}^{\infty}\left[x_{i}^{T} Q_{i} x_{i}+\lambda_{i} u_{i}^{2}\right] \mathrm{d} t
$$

is given by

$$
\bar{u}_{i}^{*}=\bar{K}_{i} x_{i},
$$

with

$$
\bar{K}_{i}=-\left(1 / \lambda_{1}\right) b_{i}^{T} R_{i},
$$

where $R_{i}$ is an $n_{i} \times n_{i}$ real symmetric positive definite matrix given as the solution of the algebraic Riccati equation,

$$
\left(1 / \lambda_{i}\right) R_{i} b_{i} b_{i}^{T} R_{i}-A_{i i}^{0 T} R_{i}-R_{i} A_{i i}^{0}-Q_{i}=0 .
$$

In (4), (6) and (7), $\lambda_{i}$ is a positive constant and $Q_{i}$ an $n_{i} \times n_{i}$ real symmetric positive definite matrix. The solution of (7) is obtained by the method suggested earlier (Singh 1970), which requires the solution of only $\left(n_{i}-1\right)$ nonlinear algebraic equations for the subsystem of order $n_{i}$. If $R_{i}$ is denoted by

$$
R_{i}=\left[\begin{array}{cccc}
r_{11} & r_{12} & \cdots & r_{1 n_{i}} \\
r_{12} & r_{22} & \cdots & r_{2 n_{i}} \\
\cdot & \cdot & \cdots & \cdot \\
\cdot & \cdot & \cdots & \cdot \\
r_{1 n_{i}} & r_{2 n_{i}} & \cdots & r_{n_{i} n_{i}}
\end{array}\right]
$$

then from (6) it follows that

$$
\bar{K}_{i}=\left[b_{i n_{i}}\left(r_{1 n_{i}} / \lambda_{i}\right)-b_{i n_{i}}\left(r_{2 n_{i}} / \lambda_{i}\right) \cdots-b_{i n_{i}} \frac{r_{n_{i} n_{i}}}{\lambda_{i}}\right] .
$$

Thus the optimal feedback control $\bar{u}_{i}^{*}$ which stabilises the interaction-free part of the $i$ th subsystem with optimal response can be generated by using (5) and (8). On incorporating the optimal feedback control, $A_{i i}^{0}$ is modified as $\vec{A}_{i i}^{0}$ and can be expressed as

$$
\vec{A}_{i i}^{0}=\left[\begin{array}{ccccccc}
0 & 1 & 0 & 0 & \cdots & . & 0 \\
0 & 0 & 1 & 0 & \cdots & . & 0 \\
. & . & . & . & \cdots & . & . \\
. & . & . & . & \cdots & . & . \\
0 & 0 & 0 & 0 & \cdots & 0 & 1 \\
d_{i 1} & d_{i 2} & d_{i 3} & d_{i 4} & \cdots & . & d_{i n_{i}}
\end{array}\right],
$$


where

$$
\begin{aligned}
d_{i 1} & =-c_{i 1}-b_{i n_{i}}\left(r_{1 n_{i}} / \lambda_{i}\right), \quad d_{i 2}=-c_{i 2}-b_{i n_{i}}\left(r_{2 n_{i}} / \lambda_{i}\right), \quad \ldots, \\
d_{i n_{i}} & =-c_{i n_{i}}-b_{i n_{i}}\left(r_{n_{i} n_{i}} / \lambda_{i}\right)
\end{aligned}
$$

\section{Aggregation-decomposition and the additional feedback controls to annul the effects of interactions}

On modifying the coefficient matrix of the interaction-free and noise-free part as above, the modified state equations corresponding to (2) are written as,

$$
\begin{aligned}
\dot{x}_{i}=\bar{A}_{i i}^{0} x_{i}+b_{i} u_{i}+a_{i} x_{l}^{T} \sum_{j=1, j \neq i, l}^{N} A_{i j}^{0} x_{j} \\
\quad+E_{i i}\left(x_{i}\right) \dot{w}_{i}+\sum_{j=1, j \neq i, l}^{N} a i x_{l}^{T} E_{i j}\left(x_{j}\right) \dot{w}_{j}
\end{aligned}
$$

By the aggregation-decomposition technique (see appendix A), the maximum possible effects of interactions and state-dependent noise can be incorporated into the coefficient matrices $\vec{A}_{i i}^{0}$ resulting in $N$ decoupled subsystems

$$
\dot{x}_{i}=\left(\bar{A}_{i i}^{0}+m_{i} I\right) x_{i}, \quad i=1,2, \ldots, N .
$$

Let

$$
\begin{aligned}
\vec{A}_{m i}^{0} \triangleq \bar{A}_{i i}^{0}+m_{i} I & \\
\Rightarrow \vec{A}_{m i}^{0}= & {\left[\begin{array}{ccccccc}
m_{i} & 1 & 0 & 0 & \cdots & . & 0 \\
0 & m_{i} & 1 & 0 & \cdots & . & 0 \\
\cdot & \cdot & \cdot & \cdot & \cdots & . & \cdot \\
\cdot & . & . & . & \cdots & . & \cdot \\
0 & 0 & 0 & 0 & \cdots & m_{i} & 1 \\
d_{i 1} & d_{i 2} & d_{i 3} & d_{i 4} & \cdots & \cdot & \left(m_{i}+d_{i n_{i}}\right)
\end{array}\right] }
\end{aligned}
$$

which on being transformed to controllable phase variable form with $n_{i} \times n_{i}$ transformation matrix $P_{i}$ is written as $A_{m i}^{0}$ given by

$$
A_{m i}^{0}=\left[\begin{array}{ccccccc}
0 & 1 & 0 & 0 & \ldots & . & 0 \\
0 & 0 & 1 & 0 & \ldots & . & 0 \\
. & . & . & . & \ldots & . & . \\
. & . & . & . & \ldots & . & . \\
0 & 0 & 0 & 0 & \ldots & 0 & 1 \\
l_{i 1} & l_{i 2} & l_{i 3} & l_{i 4} & \ldots & . & l_{i n_{i}}
\end{array}\right]
$$

The elements in the last row of $A_{m i}^{0}$ are known in terms of the elements of $\bar{A}_{m i}^{0}$ and $P_{i}$. Thus, in order to completely annul the effects of interactions and state-dependent noise terms so that the interconnected subsystems are ASwp1 with at least the same degree of 
stability and optimal response as the interaction-free and noise-free parts of the subsystems with optimal controls given by (5), the additional control gain $K_{i}$ to be introduced for the above controllable phase variable form of $A_{m i}^{0}$ to shift its eigenvalues to the same locations as of $\bar{A}_{i i}^{0}$ is given by,

$$
\underline{K}_{i}=\left[\begin{array}{llll}
d_{i 1}-l_{i 1} & d_{i 2}-l_{i 2} & \cdots & d_{i n_{i}}-l_{i n_{i}}
\end{array}\right] .
$$

Hence for the decoupled subsystems of (10) or the interconnected subsystems of (9), the additional control gain required is $K_{i} P_{i}$ to generate the additional control $u_{i}$.

$$
\Rightarrow \underline{u}_{i}=\underline{K}_{i} P_{i} x_{i} .
$$

\section{Suboptimal decentralised feedback control}

Finally, it follows that the overall state feedback gain vector $K_{i}$ in (3) for the composite system of (2) to be ASwpl with the desired degree of stability and optimal response, is given by,

$$
K_{i}=\bar{K}_{i}+\underline{K}_{i} P_{i} .
$$

In the light of the facts mentioned in earlier sections, the decentralised (stabilising) feedback controls $u_{i}$ given by equation (3) can be appropriately designated as "Suboptimal Decentralised Feedback Controls".

\section{Numerical example}

The following three stachastic bilinear interconnected second-order subsystems corresponding to (2) are considered (Siljak \& Vukcevic 1977).

$$
\begin{aligned}
\dot{x}_{1}= & {\left[\begin{array}{cc}
0 & 1 \\
0 & 0
\end{array}\right] x_{1}+\left[\begin{array}{c}
0 \\
80
\end{array}\right] u_{1}+\left[\begin{array}{c}
0 \\
-0.3
\end{array}\right] x_{2}^{T}\left[\begin{array}{ll}
0 & 0 \\
0 & 1
\end{array}\right] x_{3} } \\
& +\left[\begin{array}{cc}
0 & 0 \\
x_{11} & 0
\end{array}\right] \dot{w}_{1}+\left[\begin{array}{c}
0 \\
-0.3
\end{array}\right] x_{2}^{T}\left[\begin{array}{cc}
0 & 0 \\
x_{32} & 0
\end{array}\right] \dot{w}_{3}, \\
\dot{x}_{2}= & {\left[\begin{array}{ll}
0 & 1 \\
0 & 0
\end{array}\right] x_{2}+\left[\begin{array}{c}
0 \\
15
\end{array}\right] u_{2}+\left[\begin{array}{c}
0 \\
-0.2
\end{array}\right] x_{3}^{T}\left[\begin{array}{ll}
0 & 0 \\
0 & 1
\end{array}\right] x_{1} } \\
& +\left[\begin{array}{cc}
0 & 0 \\
x_{21} & 0
\end{array}\right] \dot{w}_{2}+\left[\begin{array}{c}
0 \\
-0.2
\end{array}\right] x_{3}^{T}\left[\begin{array}{cc}
0 & 0 \\
x_{12} & 0
\end{array}\right] \dot{w}_{1}, \\
\dot{x}_{3}= & {\left[\begin{array}{cc}
0 & 1 \\
0 & 0
\end{array}\right] x_{3}+\left[\begin{array}{c}
0 \\
10
\end{array}\right] u_{3}+\left[\begin{array}{c}
0 \\
-0.1
\end{array}\right] x_{1}^{T}\left[\begin{array}{ll}
0 & 0 \\
0 & 1
\end{array}\right] x_{2} } \\
& +\left[\begin{array}{cc}
0 & 0 \\
x_{31} & 0
\end{array}\right] \dot{w}_{3}+\left[\begin{array}{c}
0 \\
-0.1
\end{array}\right] x_{1}^{T}\left[\begin{array}{cc}
0 & 0 \\
x_{22} & 0
\end{array}\right] \dot{w}_{2} .
\end{aligned}
$$

With $Q_{1}=Q_{2}=Q_{3}=I_{4}$ and $\lambda_{1}=\lambda_{2}=\lambda_{3}=+1$ in the algebraic Riccati equations (7), the values of $R_{1}, R_{2}, R_{3}$ for the three subsystems are obtained. 
Hence corresponding to (8), the values of $\bar{K}_{1}, \bar{K}_{2}, \bar{K}_{3}$ are

$$
\begin{aligned}
& \bar{K}_{1}=\left[\begin{array}{ll}
-1.0 & -1.0124
\end{array}\right], \\
& \bar{K}_{2}=\left[\begin{array}{ll}
-1.0 & -1.0646
\end{array}\right], \\
& \bar{K}_{3}=\left[\begin{array}{ll}
-1.0 & -1.0954
\end{array}\right] .
\end{aligned}
$$

On introducing the above state feedback gains the coefficient matrices of the interactionfree and noise-free parts are modified as

$$
\begin{aligned}
& \vec{A}_{11}^{0}=\left[\begin{array}{cc}
0 & 1.0 \\
-1.0 & -1.0124
\end{array}\right], \\
& \vec{A}_{22}^{0}=\left[\begin{array}{cc}
0 & 1.0 \\
-1.0 & -1.0646
\end{array}\right], \\
& \vec{A}_{33}^{0}=\left[\begin{array}{cc}
0 & 1.0 \\
-1.0 & -1.0954
\end{array}\right] .
\end{aligned}
$$

Then using the relations in appendices A-C with $U_{1}^{0}=U_{2}^{0}=U_{3}^{0}=+0.5$ (Siljak \& Vukcevic 1977), the values of $m_{1}, m_{2}, m_{3}$ are computed as,

$$
m_{1}=0.6541, \quad m_{2}=0.1367, \quad m_{3}=0.0680 \text {. }
$$

The coefficient matrices $\vec{A}_{m 1}^{0}, \vec{A}_{m 2}^{0}, \vec{A}_{m 3}^{0}$ of the decoupled subsystems are then computed, which on being transformed to controllable phase variable form become,

$$
\begin{aligned}
& A_{m 1}^{0}=\left[\begin{array}{cc}
0.0 & 1.0 \\
-0.7656 & 0.2958
\end{array}\right], \\
& A_{m 2}^{0}=\left[\begin{array}{cc}
0.0 & 1.0 \\
-0.8731 & -0.7910
\end{array}\right], \\
& A_{m 3}^{0}=\left[\begin{array}{cc}
0.0 & 1.0 \\
-0.9301 & -0.9594
\end{array}\right]
\end{aligned}
$$

Then corresponding to (11), the additional state feedback gains to annul the effects of interactions are

$$
\begin{aligned}
& K_{1}=\left[\begin{array}{ll}
-0.2344 & -1.3082
\end{array}\right], \\
& K_{2}=\left[\begin{array}{ll}
-0.1269 & -0.2736
\end{array}\right], \\
& K_{3}=\left[\begin{array}{ll}
-0.0699 & -0.1360
\end{array}\right] .
\end{aligned}
$$

Finally, the suboptimal decentralised state feedback control gain vectors for desired stabilisation corresponding to (12) are

$$
\begin{aligned}
& K_{1}=\left[\begin{array}{ll}
-1.0780 & -0.9340
\end{array}\right] \\
& K_{2}=\left[\begin{array}{ll}
-1.0780 & -1.0240
\end{array}\right], \\
& K_{3}=\left[\begin{array}{ll}
-1.0230 & -1.0720
\end{array}\right] .
\end{aligned}
$$

\section{Conclusions}

Aggregation-decomposition technique based on algebraic Riccati equations for a class of $N$ linear interconnected subsystems developed very recently by the present authors (Patralekh 
\& Singh 1997) has been extended to a class of $N$ stochastic bilinear interconnected subsystems. This has been possible by the use of the procedure by Mahalanabis \& Purkayastha (1973). The aggregation-decomposition has been utilised for decentralised stabilisation. First the interaction-free and noise-free parts of the subsystems are stabilised by the optimal feedback controls generated by a computationally simple method (Singh 1970). Then by using the aforesaid aggregation-decomposition technique, maximum possible effects of interactions and state-dependent noise are incorporated into the coefficient matrices of the interaction-free and noise-free parts resulting in decoupled subsystems. The additional decentralised feedback control gains are introduced to annul the effects of interactions. This results in at least the same degree of stability of the stochastic interconnected subsystems with optimal response as the corresponding interaction-free and noise-free parts. Thus the desired decentralised stabilisation is achieved by Optimal Feedback Controls at subsystems levels so that the stochastic interconnected subsystems are ASwp1.

Since the procedure can be programmed on a digital coomputer, the computation of the gain parameters for decentralised stabilising controllers can be conveniently carried out even for subsystems of higher order. Implementation of the controls is quite cheap and simple because it requires only amplifiers and coefficient multipliers. Performance of the compensated system can be foreseen by simulation.

\section{Appendix A.}

The aggregation-decomposition technique based on algebraic Riccati equations reported very recently (Patralekh \& Singh 1997) is extended to the stochastic bilinear interconnected system (for details refer Mahalanabis \& Purkayastha 1973, Patralekh \& Singh 1997) as below.

In appendix $\mathrm{B}$ by taking the quadratic function $V_{i}\left(x_{i}\right)=x_{i}^{T} \bar{R}_{i} x_{i}$, where $\bar{R}_{i}$ is an $n_{i} * n_{i}$ real symmetric positive definite matrix obtained as the solution of the algebraic Riccati equation, (1B), and using the procedure of Mahalanabis \& Purkayastha (1973) based on the definition of ASwp1, the following relation has been obtained for the $i$ th subsystem of (9):

$$
\begin{aligned}
& \left.x_{i}^{T}\left(1 / \lambda_{i}\right) \bar{R}_{i} b_{i} b_{i}^{T} \bar{R}_{i}\right) x_{i}=x_{i}^{T}\left(\bar{A}_{i i}^{0} T \bar{R}_{i}+\bar{R}_{i} \bar{A}_{i i}^{0}\right) x_{i} \\
& +\sum_{j=1, j \neq i, l}^{N}\left[\operatorname{grad} V_{i}\left(x_{i}\right)\right]^{T} a_{i} x_{l}^{T} A_{i j}^{0} x_{j}+\operatorname{Tr}\left(E_{i i}\left(x_{i}\right)\left[E_{i i}\left(x_{i}\right)\right]^{T} \bar{R}_{i}\right) \\
& \quad+\sum_{j=1, j \neq i, l}^{N} \operatorname{Tr}\left(a_{i} x_{l}^{T} E_{i j}\left(x_{j}\right)\left[E_{i j}\left(x_{j}\right)\right]^{T} x_{l} a_{i}^{T} \bar{R}_{i}\right)+x_{i}^{T} Q_{i} x_{i} .
\end{aligned}
$$

As derived in appendix $\mathrm{C}$, by using the bound $\left\|x_{l}\right\| \leq U_{l}^{0}$ as in Siljak \& Vukcevic (1977), $U_{l}^{0}$ being a positive constant,

$$
\sum_{j=1, j \neq i, l}^{N}\left[\operatorname{grad} V_{i}\left(x_{i}\right)\right]^{T} a_{i} x_{l}^{T} A_{i j}^{0} x_{j} \leq \sum_{j=1, j \neq l}^{N} x_{j}^{T} D_{i j}^{0} x_{j},
$$


where $D_{i j}^{0}$ is an $n_{i} * n_{i}$ real positive definite diagonal matrix, whose elements depend on the elements of $\bar{R}_{i}, A_{i j}^{0}$, the vector $a_{i}$ and $U_{l}^{0}$. As shown in appendix $\mathrm{D}$ (by using $\left\|x_{l}\right\| \leq U_{l}^{0}$ ),

$$
\begin{aligned}
\operatorname{Tr}\left\{E_{i i}\left(x_{i}\right)\left[E_{i i}\left(x_{i}\right)\right]^{T} \bar{R}_{i}\right\} & +\sum_{j=1, j \neq i, l}^{N} \operatorname{Tr}\left\{a_{i} x_{l}^{T} E_{i j}\left(x_{j}\right)\left[E_{i j}\left(x_{j}\right)\right]^{T} x_{l} a_{i}^{T} \bar{R}_{i}\right\} \\
& \leq \sum_{j=1, j \neq l}^{N} x_{j}^{T} D_{i j}^{\prime} x_{j},
\end{aligned}
$$

where $D_{i j}^{\prime}$ is an $n_{i} * n_{i}$ real positive definite diagonal matrix.

$$
\begin{aligned}
\Rightarrow x_{i}^{T}\left(\left(1 / \lambda_{i}\right) \bar{R}_{i} b_{i} b_{i}^{T} \bar{R}_{i}\right) x_{i} \leq & x_{i}^{T}\left(\bar{A}_{i i}^{0} T \bar{R}_{i}+\bar{R}_{i} \bar{A}_{i i}^{0}\right) x_{i} \\
& +\sum_{j=1, j \neq l}^{N} x_{j}^{T} D_{i j} x_{j}+x_{i}^{T} Q_{i} x_{i},
\end{aligned}
$$

where $D_{i j}=D_{i j}^{0}+D_{i j}^{\prime}$, and is an $n_{i} \times n_{i}$ real positive definite diagonal matrix. Hence the following inequality is obtained for subsystems of (9),

$$
\begin{aligned}
\sum_{i=1}^{N} x_{i}^{T}\left(\left(1 / \lambda_{i}\right) \bar{R}_{i} b_{i} b_{i}^{T} \bar{R}_{i}\right) x_{i} \leq & \sum_{i=1}^{N} x_{i}^{T}\left(\bar{A}_{i i}^{0} T \bar{R}_{i}+\bar{R}_{i} \bar{A}_{i i}\right) x_{i} \\
& +\sum_{i=1}^{N} \sum_{j=1, j \neq 1}^{N} x_{j}^{T} D_{i j} x_{j}+\sum_{i=1}^{N} x_{i}^{T} Q_{i} x_{i} .
\end{aligned}
$$

It can be proved that (appendix E),

$$
\sum_{i=1}^{N} \sum_{j=1, j \neq l}^{N} x_{j}^{T} D_{i j} x_{j} \leq \sum_{i=1}^{N} 2 m_{i} x_{i}^{T} \bar{R}_{i} x_{i},
$$

where $m_{i}$ is real and positive,

$$
\begin{aligned}
\Rightarrow \sum_{i=1}^{N} x_{i}^{T}\left(\left(1 / \lambda_{i}\right) \bar{R}_{i} b_{i} b_{i}^{T} \bar{R}_{i}\right) x_{i} \leq & \sum_{i=1}^{N} x_{i}^{T}\left(\bar{A}_{i i}^{0} T \bar{R}_{i}+\bar{R}_{i} \bar{A}_{i i}^{0}\right) x_{i} \\
& +\sum_{i=1}^{N} 2 m_{i} x_{i}^{T} \bar{R}_{i} x_{i}+\sum_{i=1}^{N} x_{i}^{T} Q_{i} x_{i} .
\end{aligned}
$$

The above inequality can be replaced by an equation by replacing $\bar{R}_{i}$ by another $n_{i} \times n_{i}$ real symmetric positive definite matrix $R_{i}$. The resulting equation will be reduced to the following form (Patralekh \& Singh 1997):

$$
\left(1 / \lambda_{i}\right) \underline{R}_{i} b_{i} b_{i}^{T} \underline{R}_{i}=\left(\bar{A}_{i i}^{0}+m_{i} I\right)^{T} \underline{R}_{i}+\underline{R}_{i}\left(\bar{A}_{i i}^{0}+m_{i} I\right)+Q_{i},
$$

which are algebraic Riccati equations for the systems,

$$
\dot{x}_{i}=\left(\bar{A}_{i i}^{0}+m_{i} I\right) x_{i}, \quad i=1,2, \ldots, N .
$$

Thus the maximum possible effects of the interactions have been incorporated into the coefficient matrices of the interaction-free parts of the subsystems of (9) resulting in $N$ decoupled subsystems of (A1). 


\section{Appendix B.}

Consider the subsystem of (9),

$$
\begin{aligned}
\dot{x}_{i}= & \bar{A}_{i i}^{0} x_{i}+b_{i} u_{i}+a_{i} x_{l}^{T} \sum_{j=1, j \neq i, l}^{N} A_{i j}^{0} x_{j} \\
& +E_{i i}\left(x_{i}\right) \dot{w}_{i}+\sum_{j=1, j \neq i, l}^{N} a_{i} x_{l}^{T} E_{i j}\left(x_{j}\right) \dot{w}_{j}, \quad i=1,2, \ldots, N .
\end{aligned}
$$

Then the algebraic Riccati equation for the interaction-free and noise-free part of (9) is written by replacing $R_{i}$ by $\bar{R}_{i}$ and $A_{i i}^{0}$ by $\bar{A}_{i i}^{0}$ in (7) as,

$$
\left(1 / \lambda_{i}\right) \bar{R}_{i} b_{i} b_{i}^{T} \bar{R}_{i}=\bar{A}_{i i}^{0} T \bar{R}_{i}+\bar{R}_{i} \bar{A}_{i i}^{0}+Q_{i} .
$$

Hence for the interaction-free and noise-free part of (9)

$$
\left.x_{i}^{T}\left(1 / \lambda_{i}\right) \bar{R}_{i} b_{i} b_{i}^{T} \bar{R}_{i}\right) x_{i}=x_{i}^{T}\left(\bar{A}_{i i}^{0} T \bar{R}_{i}+\bar{R}_{i} \bar{A}_{i i}^{0}\right) x_{i}+x_{i}^{T} Q_{i} x_{i} .
$$

Let the quadratic function $V_{i}\left(x_{i}\right)=x_{i}^{T} \bar{R}_{i} x_{i}$, where $\bar{R}_{i}$ is an $n_{i} \times n_{i}$ real symmetric positive definite matrix obtained as the solution of the algebraic Riccati equation (B1) which implies that $V_{i}\left(x_{i}\right)$ is positive definite.

Refering to Mahalanabis \& Purkayastha (1973), for the interaction-free and noise-free autonomous part of (9),

$$
\begin{aligned}
& L V_{i}\left(x_{i}\right)=\left(\bar{A}_{i i}^{0} x_{i}\right)^{T} \nabla x_{i} V_{i}\left(x_{i}\right), \\
& \quad \Rightarrow L V_{i}\left(x_{i}\right)=x_{i}^{T}\left(\bar{A}_{i i}^{0} T \bar{R}_{i}+\bar{R}_{i} \bar{A}_{i i}^{0}\right) x_{i}, \\
& \quad \Rightarrow x_{i}^{T}\left(\left(1 / \lambda_{i}\right) \bar{R}_{i} b_{i} b_{i}^{T} \bar{R}_{i}\right) x_{i}=L V_{i}\left(x_{i}\right)+x_{i}^{T} Q_{i} x_{i} .
\end{aligned}
$$

Using the procedure of the Mahalanabis \& Purkayastha (1973) based on the definition of ASwp1, for the subsystem (9) we get,

$$
\begin{aligned}
L V_{i}\left(x_{i}\right)= & \left(\bar{A}_{i i}^{0} x_{i}\right)^{T} \nabla_{x_{i}} V_{i}\left(x_{i}\right)+\sum_{j=1, j \neq i, l}^{N}\left(a_{i} x_{l}^{T} A_{i j}^{0} x_{j}\right)^{T} \nabla_{x_{i}} V_{i}\left(x_{i}\right) \\
& +(1 / 2) \operatorname{Tr}\left\{E_{i i}\left(x_{i}\right)\left[E_{i i}\left(x_{i}\right)\right]^{T} \nabla_{x_{i}, x_{i}} V_{i}\left(x_{i}\right)\right\} \\
& +\sum_{j=1, j \neq i, l}^{N}(1 / 2) \operatorname{Tr}\left\{a_{i} x_{l}^{T} E_{i j}\left(x_{j}\right)\left[a_{i} x_{l}^{T} E_{i j}\left(x_{j}\right)\right]^{T} \nabla_{x_{i}, x_{i}} V_{i}\left(x_{i}\right)\right\}, \\
\Rightarrow L V_{i}\left(x_{i}\right)= & x_{i}^{T}\left(\bar{A}_{i i}^{0} T \bar{R}_{i}+\bar{R}_{i} \bar{A}_{i i}^{0}\right) x_{i}+\sum_{j=1, j \neq i, l}^{N}\left[\operatorname{grad} V_{i}\left(x_{i}\right)\right]^{T} a_{i} x_{i}^{T} A_{i j}^{0} x_{j} \\
& +\operatorname{Tr}\left\{E_{i i}\left(x_{i}\right)\left[E_{i i}\left(x_{i}\right)\right]^{T} \bar{R}_{i}\right\} \\
& +\sum_{j=1, j \neq i, l}^{N} \operatorname{Tr}\left\{a_{i} x_{l}^{T} E_{i j}\left(x_{j}\right)\left[E_{i j}\left(x_{j}\right)\right]^{T} x_{l} a_{i}^{T} \bar{R}_{i}\right\} .
\end{aligned}
$$


Hence for the subsystem (9) it follows that

$$
\begin{aligned}
& x_{i}^{T}\left(\left(1 / \lambda_{i}\right) \bar{R}_{i} b_{i} b_{i}^{T} \bar{R}_{i}\right) x_{i}=x_{i}^{T}\left(\bar{A}_{i i}^{0} T \bar{R}_{i}+\bar{R}_{i} \vec{A}_{i i}^{0}\right) x_{i} \\
& +\sum_{j=1, j \neq i, l}^{N}\left[\operatorname{grad} V_{i}\left(x_{i}\right)\right]^{T} a_{i} x_{l}^{T} A_{i j}^{0} x_{j}+\operatorname{Tr}\left\{E_{i i}\left(x_{i}\right)\left[E_{i i}\left(x_{i}\right)\right]^{T} \bar{R}_{i}\right\} \\
& \quad+\sum_{j=1, j \neq i, l}^{N} \operatorname{Tr}\left\{a_{i} x_{l}^{T} E_{i j}\left(x_{j}\right)\left[E_{i j}\left(x_{j}\right)\right]^{T} x_{l} a_{i}^{T} \bar{R}_{i}\right\}+x_{i}^{T} Q_{i} x_{i}
\end{aligned}
$$

\section{Appendix C.}

To derive the inequality

$$
\sum_{j=1, j \neq i, l}^{N}\left[\operatorname{grad} V_{i}\left(x_{i}\right)\right]^{T} a_{i} x_{l}^{T} A_{i j}^{0} x_{j} \leq \sum_{j=1, j \neq l}^{N} x_{j}^{T} D_{i j}^{0} x_{j},
$$

where $D_{i j}^{0}$ is an $n_{i} \times n_{i}$ real positive definite diagonal matrix, it is noted that the term $\left[\operatorname{grad} V_{i}\left(x_{i}\right)\right]^{T} a_{i} x_{l}^{T} A_{i j}^{0} x_{j}$ can be expressed as follows,

$$
\begin{aligned}
{\left[\operatorname{grad} V_{i}\left(x_{i}\right)\right]^{T} a_{i} x_{l}^{T} A_{i j}^{0} x_{j}=} & {\left[\frac{\partial V_{i}\left(x_{i}\right)}{\partial x_{i 1}} \cdots \frac{\partial V_{i}\left(x_{i}\right)}{\partial x_{i n_{i}}}\right]\left[\begin{array}{c}
a_{i 1} \\
\vdots \\
a_{i n_{i}}
\end{array}\right]\left[x_{l 1} \cdots x_{l n_{i}}\right] } \\
& \times\left[\begin{array}{cc}
a_{i j 11} x_{j 1}+\cdots+a_{i j 1 n_{j}} x_{j n_{j}} \\
\cdot \\
\cdots \\
\cdots \\
\cdots \\
\cdot \\
a_{i j n_{i} 1 x_{j 1}+\cdots+a_{i j n_{i} n_{j}} x_{j n_{j}}}+\cdots
\end{array}\right]
\end{aligned}
$$

If the multiplications are carried out after explicitly evaluating the partial derivatives, we get terms of the form

$$
\begin{aligned}
& 2\left(\sum_{v=1}^{N} r_{i v w} a_{i v}\right) a_{i j v^{\prime} w^{\prime}} x_{i w} x_{j w^{\prime}} x_{l_{s}} ; \quad i, j=1,2, \ldots, N \\
& \quad v, w, v^{\prime}, s=1,2, \ldots, n_{i} ; \quad w^{\prime}=1,2, \ldots, n_{j}
\end{aligned}
$$

where $x_{l_{s}} \leq\left\|x_{l}\right\|$.

As in Siljak \& Vukcevic (1977), it is assumed that $\left\|x_{l}\right\|$ has the bound,

$$
\left\|x_{l}\right\| \leq U_{l}^{0}, \quad U_{l}^{0} \text { being a positive constant. }
$$

Hence, the terms of the above form can be bounded as below,

$$
\begin{aligned}
& 2\left(\sum_{v=1}^{n_{i}} r_{i v w} a_{i v}\right) a_{i j v^{\prime} w^{\prime}} x_{i w} x_{j w^{\prime}} x_{l_{s}} \\
& \leq\left\|\sum_{v=1}^{n_{i}} r_{i v w} a_{i v}\right\|\left|a_{i j v^{\prime} w^{\prime}}\right|\left(x_{i w}^{2}+x_{j w^{\prime}}^{2}\right) U_{l}^{0},
\end{aligned}
$$


where $r_{i v w}$ represents the $v$, wth element of the matrix $\bar{R}_{i}$ and $a_{i j v^{\prime} w^{\prime}}$ the $v^{\prime}, w^{\prime}$ th element of the matrix $A_{i j}^{0}$.

Using this, it is possible to get the inequality

$$
\left[\operatorname{grad} V_{i}\left(x_{i}\right)\right]^{T} a_{i} x_{l}^{T} A_{i j}^{0} x_{j} \leq x_{i}^{T} D_{i i} x_{i}+x_{j}^{T} D_{i j}^{0} x_{j},
$$

where $D_{i i}$ and $D_{i j}^{0}$ are diagonal matrices whose elements are real non-negative numbers depending upon the elements of the matrices $A_{i j}^{0}, \bar{R}_{i}$, the vector $a_{i}$ and $U_{l}^{0}$.

Hence the following inequality must hold good.

$$
\begin{aligned}
& \left.\sum_{j=1, j \neq i, l}^{N}\left[\operatorname{grad} V_{i}\left(x_{i}\right)\right]^{T} a_{i} x_{l}^{T} A_{i j}^{0} x_{j} \leq \sum_{j=1, j \neq i, l}^{N} x_{i}^{T} D_{i i} x_{i}+x_{j}^{T} D_{i j}^{0} x_{j}\right) \\
\Rightarrow & \sum_{j=1, j \neq i, l}^{N}\left[\operatorname{grad} V_{i}\left(x_{i}\right)\right]^{T} a_{i} x_{l}^{T} A_{i j}^{0} x_{j} \leq x_{i}^{T}\left\{(N-2) D_{i i}\right\} x_{i} \\
& +\sum_{j=1, j \neq i, l}^{N} x_{j}^{T} D_{i j}^{0} x_{j} \\
\Rightarrow & \sum_{j=1, j \neq i, l}^{N}\left[\operatorname{grad} V_{i}\left(x_{i}\right)\right]^{T} a_{i} x_{l}^{T} A_{i j}^{0} x_{j} \leq x_{i}^{T} D_{i i}^{0} x_{i}+\sum_{j=1, j \neq i, l}^{N} x_{j}^{T} D_{i j}^{0} x_{j} \\
\Rightarrow & \sum_{j=1, j \neq i, l}^{N}\left[\operatorname{grad} V_{i}\left(x_{i}\right)\right]^{T} a_{i} x_{l}^{T} A_{i j}^{0} x_{j} \leq \sum_{j=1, j \neq l}^{N} x_{j}^{T} D_{i j}^{0} x_{j},
\end{aligned}
$$

where $D_{i i}^{0}=(N-2) D_{i i}$ and $D_{i j}^{0}$ is an $n_{i} \times n_{i}$ real positive definite diagonal matrix.

\section{Appendix D.}

In order to derive

$$
\begin{aligned}
& \operatorname{Tr}\left\{E_{i i}\left(x_{i}\right)\left[E_{i i}\left(x_{i}\right)\right]^{T} \bar{R}_{i}\right\}+\sum_{j=1, j \neq i, l}^{N} \operatorname{Tr}\left\{a_{i} x_{l}^{T} E_{i j}\left(x_{j}\right)\left[E_{i j}\left(x_{j}\right)\right]^{T} x_{l} a_{i}^{T} \bar{R}_{i}\right\} \\
& \leq \sum_{j=1, j \neq l}^{N} x_{j}^{T} D_{i j}^{\prime} x_{j},
\end{aligned}
$$

first it will be shown that

$$
\begin{aligned}
& \operatorname{Tr}\left\{E_{i i}\left(x_{i}\right)\left[E_{i i}\left(x_{i}\right)\right]^{T} \bar{R}_{i}\right\} \leq x_{i}^{T} D_{i i}^{\prime} x_{i}, \\
& \operatorname{Tr}\left\{a_{i} x_{l}^{T} E_{i j}\left(x_{j}\right)\left[E_{i j}\left(x_{j}\right)\right]^{T} x_{l} a_{i}^{T} \bar{R}_{i}\right\} \leq x_{j}^{T} D_{i j}^{\prime} x_{j} .
\end{aligned}
$$

In order to prove (D1), it is noted that $E_{i i}\left(x_{i}\right)$ has the form,

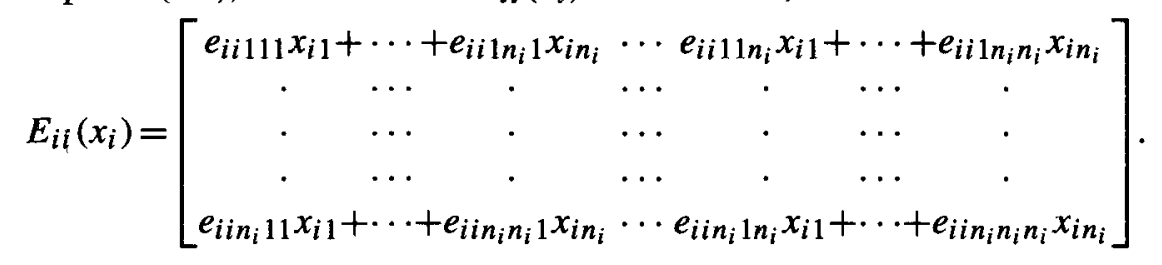


On multiplying $E_{i i}\left(x_{i}\right),\left[E_{i i}\left(x_{i}\right)\right]^{T}, R_{i}$ and evaluating the trace of the product we get terms of the form $h_{i v} x_{i v}^{2}, h_{i l v} x_{i l} x_{i v}$. The coefficients $h_{i v}, h_{i l v}$ depend on the scalars $e_{i i w v l}$ in the elements of the matrix $E_{i i}\left(x_{i}\right)$ and the elements $r_{i l w}$ of the matrix $\bar{R}_{i}$. The terms $h_{i v} x_{i v}^{2}$ and $h_{i l v} x_{i l} x_{i v}$ can be bounded as below,

$$
\begin{aligned}
h_{i v} x_{i v}^{2} & \leq\left|h_{i v}\right| x_{i v}^{2}, \\
h_{i l v} x_{i l} x_{i v} & \leq(1 / 2)\left|h_{i l v}\right|\left(x_{i l}^{2}+x_{i v}^{2}\right) .
\end{aligned}
$$

Using these it is possible to get the inequality

$$
\operatorname{Tr}\left\{E_{i i}\left(x_{i}\right)\left[E_{i i}\left(x_{i}\right)\right]^{T} R_{i}\right\} \leq x_{i}^{T} D_{i i}^{\prime} x_{i},
$$

where $D_{i i}^{\prime}$ is an $n_{i} \times n_{i}$ real positive definite diagonal matrix.

In order to derive (D2), it is noted that $E_{i j}\left(x_{j}\right)$ has the following form

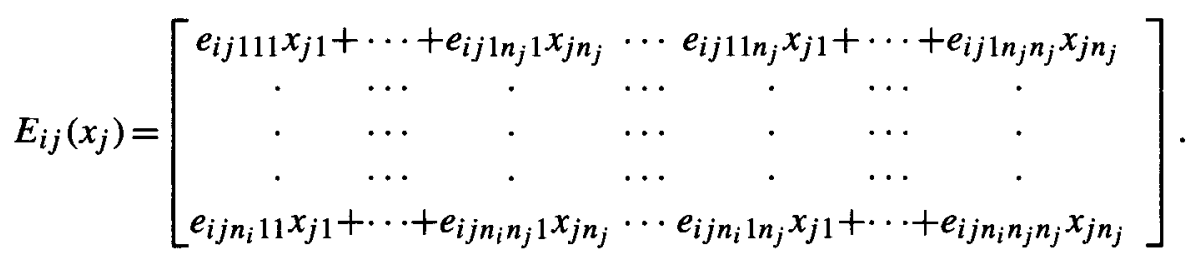

Then on evaluating the trace of the product of the factors within the middle brackets of the left hand side of (D2), one gets terms of the form $g_{j v^{\prime}} x_{j v^{\prime}}^{2} x_{l v^{\prime}}^{2}, g_{j w^{\prime} v^{\prime}} x_{j w^{\prime}} x_{j v^{\prime}} x_{l w^{\prime}} x_{l v^{\prime}}$.

Coefficients $g_{j v^{\prime}}, g_{j w^{\prime} v^{\prime}}$ depend on the coefficients $e_{i j w v^{\prime} w^{\prime}}$ in the elements of the matrix $E_{i j}\left(x_{j}\right)$, the elements $r_{i l w}$ of the matrix $\bar{R}_{i}$, the elements of the vector $a_{i}$ and $U_{l}^{0}$. The above terms can be bounded as below,

$$
\begin{aligned}
& g_{j v^{\prime}} x_{j v^{\prime}}^{2} x_{l v^{\prime}}^{2} \leq\left|g_{j v^{\prime}}\right| U_{l}^{02} x_{j v^{\prime}}^{2}, \\
& g_{j w^{\prime} v^{\prime}} x_{j w^{\prime}} x_{j v^{\prime}} x_{l w^{\prime}} x_{l v^{\prime}} \leq(1 / 2)\left|g_{j w^{\prime} v^{\prime}}\right| U_{l}^{02}\left(x_{j w^{\prime}}^{2}+x_{j v^{\prime}}^{2}\right) .
\end{aligned}
$$

with $\left\|x_{l}\right\| \leq U_{l}^{0}$, where $U_{l}^{0}$ is a positive constant.

Using this, it is possible to get the inequality

$$
\operatorname{Tr}\left\{a_{i} x_{l}^{T} E_{i j}\left(x_{j}\right)\left[E_{i j}\left(x_{j}\right)\right]^{T} x_{i} a_{i}^{T} \bar{R}_{i}\right\} \leq x_{j}^{T} D_{i j}^{\prime} x_{j} .
$$

Hence it follows that

$$
\begin{aligned}
& \operatorname{Tr}\left\{E_{i i}\left(x_{i}\right)\left[E_{i i}\left(x_{i}\right)\right]^{T} \bar{R}_{i}\right\}+\sum_{j=1, j \neq i, l}^{N} \operatorname{Tr}\left\{a_{i} x_{l}^{T} E_{i j}\left(x_{j}\right)\left[E_{i j}\left(x_{j}\right)\right]^{T} x_{l} a_{i}^{T} \bar{R}_{i}\right\} \\
& \quad \leq \sum_{j=1, j \neq l}^{N} x_{j}^{T} D_{i j}^{\prime} x_{j},
\end{aligned}
$$

where $D_{i j}$ is an $n_{i} \times n_{i}$ real positive definite diagonal matrix.

\section{Appendix E.}

In order to prove that 


$$
\sum_{i=1}^{N} \sum_{j=1, j \neq l}^{N} x_{j}^{T} D_{i j} x_{j} \leq \sum_{i=1}^{N} 2 m_{i} x_{i}^{T} \bar{R}_{i} x_{i},
$$

we proceed as follows,

$$
\begin{aligned}
& \sum_{i=1}^{N} \sum_{j=1, j \neq l}^{N} x_{j}^{T} D_{i j} x_{j}=\sum_{i=1}^{N} x_{i}^{T}\left(\sum_{j=1, j \neq l}^{N} D_{j i}\right) x_{i} \\
& \Rightarrow \sum_{i=1}^{N} \sum_{j=1, j \neq l}^{N} x_{j}^{T} D_{i j} x_{j}=\sum_{i=1}^{N} x_{i}^{T} M_{i} x_{i},
\end{aligned}
$$

where $M_{i}=\sum_{j=1, j \neq l}^{N} D_{j i} \Rightarrow M_{i}$ is an $n_{i} \times n_{i}$ real positive definite diagonal matrix.

Now an $n_{i} \times n_{i}$ real positive definite diagonal (nonsingular) matrix $G_{i}$ is chosen such that

$$
G_{i} G_{i}^{T}=M_{i}
$$

Then since $\bar{R}_{i}$ also is a real symmetric positive definite matrix, the $n_{i} \times n_{i}$ matrix $S_{i}=$ $G_{i}^{-1} \bar{R}_{i}\left(G_{i}^{-1}\right)^{T}$ is also a real symmetric positive definite matrix. It is known that for a real symmetric positive definite matrix $S_{i}$, the following inequality must hold good,

$$
I \leq\left(1 / \beta_{i}\right) S_{i},
$$

where $\beta_{i}$ is the minimum eigenvalue of $S_{i}$ and is real and positive,

$$
\begin{aligned}
& \Rightarrow I \leq\left(1 / \beta_{i}\right) G_{i}^{-1} \bar{R}_{i}\left(G_{i}^{-1}\right)^{T}, \\
& \Rightarrow G_{i} G_{i}^{T} \leq\left(1 / \beta_{i}\right) \bar{R}_{i}, \\
& \Rightarrow M_{i} \leq\left(1 / \beta_{i}\right) \bar{R}_{i}, \\
& \Rightarrow x_{i}^{T} M_{i} x_{i} \leq\left(1 / \beta_{i}\right)\left(x_{i}^{T} \bar{R}_{i} x_{i}\right), \\
& \Rightarrow x_{i}^{T} M_{i} x_{i} \leq 2 m_{i}\left(x_{i}^{T} \bar{R}_{i} x_{i}\right),
\end{aligned}
$$

where $m_{i}=1 /\left(2 \beta_{i}\right)$ is also real and positive,

$$
\Rightarrow \sum_{i=1}^{N} \sum_{j=1, j \neq l}^{N} x_{j}^{T} D_{i j} x_{j} \leq \sum_{i=1}^{N} 2 m_{i} x_{i}^{T} \bar{R}_{i} x_{i} .
$$

\section{References}

Siljak D D, Vukcevik M B 1977 Decentrally stabilizable linear and bilinear large-scale systems. Int. J. Control 26: 289-305

Patralekh K, Singh R 1997 Stabilisation of a class of large scale linear system by suboptimal decentralised feedback control. Inst. Eng. (India), Electron. Telecommun. Eng. Div. 78: 28-33

Mahalanabis A K, Purkayastha S 1973 Frequency-domain criteria for stability of a class of nonlinear stochastic systems. IEEE Trans. Autom. Contol AC-18: 266-270

Singh R 1970 Optimal feedback control of linear time-invariant system with quadratic criterion. Inst. Eng. (India), Electron. Telecommun. Eng. Div. 51: 52-55 